\title{
Long-term effects of evodiamine on expressions of lipogenesis and lipolysis genes in mouse adipose and liver tissues
}

\author{
D.F. Jiang ${ }^{1,2 *}$, W.T. Li ${ }^{2 *}$, H.L. Yang ${ }^{1 *}$, Z.Z. Zhang ${ }^{1 *}$, D. Chen ${ }^{1}$ and \\ C. Sun ${ }^{1}$ \\ ${ }^{1}$ College of Animal Science and Technology, Northwest A\&F University, \\ Yangling, Shaanxi, China \\ ${ }^{2}$ Zhengzhou College of Animal Husbandry Engineering, Zhengzhou, \\ Henan, China \\ *These authors contributed equally to this study. \\ Corresponding author: C. Sun \\ E-mail: sunchao2775@163.com
}

Genet. Mol. Res. 13 (1): 1038-1046 (2014)

Received January 14, 2013

Accepted July 5, 2013

Published February 20, 2014

DOI http://dx.doi.org/10.4238/2014.February.20.5

\begin{abstract}
Evodiamine, the major alkaloid component isolated from the fruit of dried, unripened Evodia rutaecarpa Bentham, affects the plasma levels of cholecystokinin and various biological events such as gastric emptying and gastrointestinal transit; these effects of evodiamine were previously investigated in male rats. In this study, we aimed to investigate the effects of evodiamine on average daily weight gain, rectal temperature, and expressions of genes involved in lipid metabolism in liver and adipose tissues. Evodiamine was added as a supplement, comprising $0.02,0.04$, and $0.06 \%$ of the diet fed to mice for 1, 2, 3, and 4 weeks. Results showed that average daily weight gain and rectal temperature decreased significantly over time in a dosedependent manner. Evodiamine changed expressions of the peroxisome proliferator-activated receptor- $\gamma$ (PPAR $\gamma$ ) in mouse adipose and liver tissues in time- and dose-dependent manners. We found that evodiamine
\end{abstract}


decreased mRNA expression of the sterol-regulatory element binding protein (SREBP-1c) and fatty acid synthase in adipose tissue. In addition, evodiamine increased expressions of hormone-sensitive lipase in both liver and adipose tissues. Interestingly, evodiamine increased the expression of triglyceride hydrolase only in adipose tissue. In conclusion, evodiamine could influence lipid metabolism through regulation of the expressions of its key genes, as well as reduce body heat and body weight.

Key words: Evodiamine; Long-term effect; Gene expression; Adipose; Metabolism

\section{INTRODUCTION}

The adipocyte plays a critical role in energy balance. Adipose tissue growth involves an increase in adipocyte size and the formation of new adipocytes from precursor cells. This is accompanied by a dramatic increase in expressions of adipocyte genes, including peroxisome proliferator-activated receptor- $\gamma$ (PPAR $\gamma$ ), fatty acid synthase (FAS), sterol-regulatory element binding protein (SREBP), hormone-sensitive lipase (HSL), and triglyceride hydrolase (TGH) genes (Fajas et al., 1998). Evodiamine is the main active alkali of the traditional Chinese medicinal plant, Evodia rutaecarpa Benth. It is well known for its anti-tumor, anti-inflammatory, and weight reduction effects. Moreover, it has been used in the treatment of abdominal pain, diarrhea, nausea, and for many other pharmacological effects (Ogasawara et al., 2002; Shyu et al., 2006; Ko et al., 2007; Wang et al., 2008). In addition, evodiamine can induce the lipolysis rate in mice and enhance specific binding of guanosine diphosphate to mitochondria in interscapular brown adipose tissue, where it increases heat dissipation and promotes energy consumption. Evodiamine can also reduce perirenal, epididymal, and hepatic fat depositions, subsequently reducing levels of free fatty acids, triglycerides, and cholesterol in human bodies. All of these effects are important to prevent fat accumulation around internal organs and to control weight gain (Kobayashi et al., 2001). However, despite this evidence, little is known about how evodiamine might regulate expressions of genes involved in lipid metabolism. In this study, we provide the first report on the molecular mechanism involved in evodiaminemediated regulation of fat deposition.

\section{MATERIAL AND METHODS}

\section{Animals}

Two-week-old male Kumming mice were obtained from the Fourth Military Medical University (Xi'an, China), and housed in standard cages in a climate-controlled room with an ambient temperature of $25^{\circ} \mathrm{C}$ and a 12-h light/dark cycle. All animals were allowed free access to water and food during the acclimatization period of 7 days. Mice were then randomly divided into four groups, consisting of 20 animals each, including the control group (basal diet) and experimental groups, whose diets were supplemented with evodiamine (purity $>98.0 \%$, Guanyu Biotechnology Inc., Xi' an, China) in doses of $0.02,0.04$, and $0.06 \%$ of their 
diets. Following diet treatments, mice in all groups were immediately weighed, their rectal temperatures were recorded, and were then killed by decapitation. Subcutaneous adipose and liver tissues were rapidly separated, immediately frozen in liquid nitrogen, and stored at $-80^{\circ} \mathrm{C}$ until RNA extraction.

\section{RNA extraction and cDNA synthesis}

Total RNA was extracted from adipose and liver tissues using the Trizol reagent (Invitrogen, USA). First-strand cDNA was prepared with the Revert-Aid ${ }^{\mathrm{TM}}$ First-Strand cDNA Synthesis Kit (Fermentas, Shenzhen, China). Primers of the $\beta$-actin, PPAR $\gamma$, SREBP-1c, FAS, HSL, and TGH genes, and polymerase chain reaction (PCR) conditions are summarized in Table 1. The $25-\mu \mathrm{L}$ PCR mixture contained $1 \mu \mathrm{L}$ tissue-specific cDNA, $2.5 \mu \mathrm{L} 25 \mathrm{mM} \mathrm{MgCl}_{2}$, $0.2 \mu \mathrm{L}$ Taq DNA polymerase (Fermentas), $2.5 \mu \mathrm{L} 2.5 \mathrm{mM}$ dNTPs, $2.5 \mu \mathrm{L} 10 \mathrm{X}$ buffer, and 1 $\mu \mathrm{L} 10 \mu \mathrm{M}$ of each primer. Products were examined by agarose gel electrophoresis.

\section{Real-time PCR}

We measured expressions of PPAR $\gamma$, SREBP-1c, FAS, HSL, and TGH genes using semi-quantitative PCR; the $\beta$-actin gene was used as the internal control. The primers and PCR amplification conditions are listed in Table 1. The PCR products were examined by agarose gel electrophoresis.

\begin{tabular}{llc}
\multicolumn{2}{c}{ Table 1. Sequence of primers and melting temperature $(\mathrm{Tm})}$. & Tm $\left({ }^{\circ} \mathrm{C}\right)$ \\
\hline Gene and accession No. & Primer sequences $\left(5^{\prime}-3^{\prime}\right)$ & 53.8 \\
\hline$\beta$-actin (NM_007393.2) & Forward: ACTGCCGCATCCTCTTCCTC & 52.1 \\
PPAR $\gamma($ U01664) & Reverse: CTCCTGCTTGCTGATCCACATC & 59.7 \\
SREBP (AB017337) & Forward: ACCACTCGCATTCCTTTAC & 55.9 \\
FAS (BC046513) & Feverse: CCACAGACTCGGCACTCAAT & 5 \\
HSL (NM_010719) & Reverse: ATGGAGACATCGCAAACAAGC & 57.9 \\
TGH (NM_053200) & Forward: AGTGTCCACCACACACGCATC & 53.3 \\
& Reverse: GATGCCGTCAGGTTTCAG & 5 \\
\hline
\end{tabular}

Data are reported as means \pm SE. Statistical analysis was performed using the SPSS 13.0 program. One-way analysis of variance and Fisher's least significant difference procedure were employed to determine the significance of differences, and Pearson's correlation analysis was used for correlation studies.

\section{RESULTS}

\section{Evodiamine affected average daily weight gain (ADG) of mice}

To investigate the effect of evodiamine on body weight, the ADG of mice was recorded. Every week before mice were killed, the body weights $\left(\mathrm{BW}_{\mathrm{n}}\right)$ were determined, 
and ADG was obtained by the formula: $\left(\mathrm{BW}_{\mathrm{n}}-\mathrm{BW}_{(\mathrm{n}-1)}\right) / 7$. The results revealed that ADG decreased significantly with increasing time and evodiamine dose $(\mathrm{P}<0.01$; Figure $1 \mathrm{~A})$.
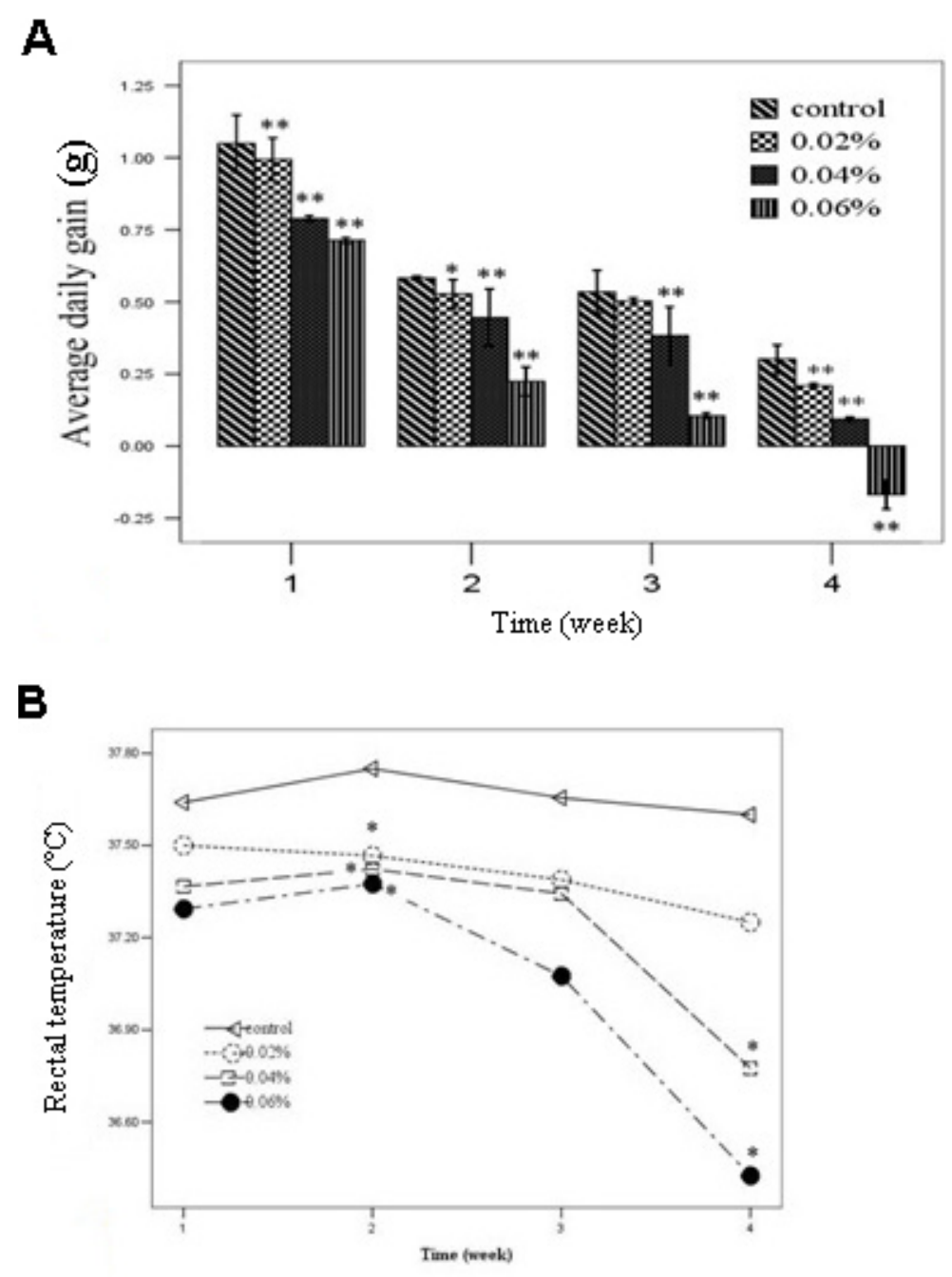

Figure 1. Effect of evodialmine on mice average daily weight gain $(\mathbf{A})$ and on the rectal temperature $(\mathbf{B}) .{ }^{* *} \mathrm{P}<$ 0.01 and $* \mathrm{P}<0.05$.

\section{Effect of evodiamine on rectal temperature}

We investigated the effect of evodiamine on rectal temperature in mice. Rectal temperatures were measured at 1,2,3, and 4 weeks in food-fasted mice. Figure 1B shows that the average rectal temperatures of experimental groups were all lower than those of the control group, which also showed time- and dose-dependent effects. 


\section{Effect of evodiamine on PPAR $\gamma$, SREBP-1c, and FAS gene expressions}

Evodiamine played a significant role in regulating the expression of genes involved in lipogenesis. We measured expressions of the PPAR $\gamma$ and SREBP-1c genes, which are representative genes of the adipocyte differentiation process. As shown in Figure 2A and B, evodiamine changed expressions of the PPAR $\gamma$ gene in both liver and adipose tissues of mice. Different doses had different effects at different times. SREBP-1c gene expression decreased in both liver and adipose tissues, but the effect was more significant in adipose tissues (Figure $3 \mathrm{~A}$ and $\mathrm{B}$ ). One rate-limiting factor for fat deposition in animal is FAS. Our results showed that within 3 weeks, supplementation with $0.06 \%$ evodiamine could decrease expression of the FAS gene in the liver; when supplemented with $0.04 \%$, FAS expression increased before 2 weeks and then decreased; when supplemented with $0.02 \%$, FAS expression showed no significance compared with control. As a whole, there is no obvious regularity in liver (Figure 4A). By contrast, in the subcutaneous adipose tissue, evodiamine drastically reduced FAS mRNA expression in a time- and dose-dependent manner $(\mathrm{P}<0.01$, Figure $4 \mathrm{~B})$.

A

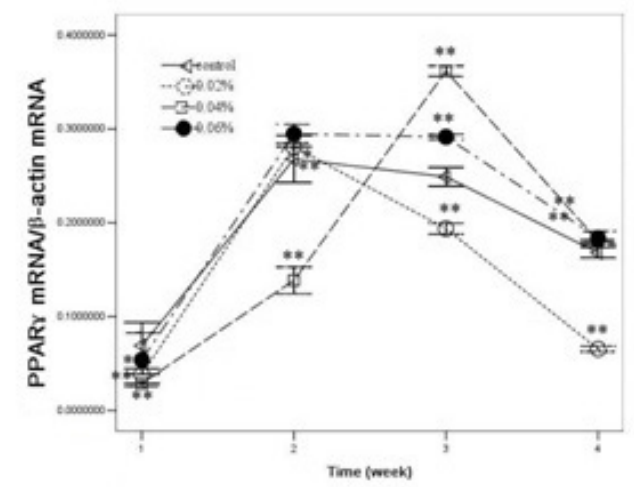

B

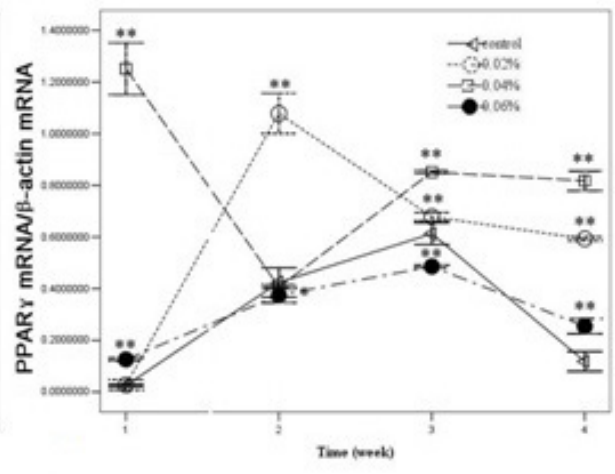

Figure 2. Evodiamine affects PPAR $\gamma$ mRNA expression in mouse liver $(\mathbf{A})$ and adipose tissues $(\mathbf{B}) .{ }^{* *} \mathrm{P}<0.01$.

A

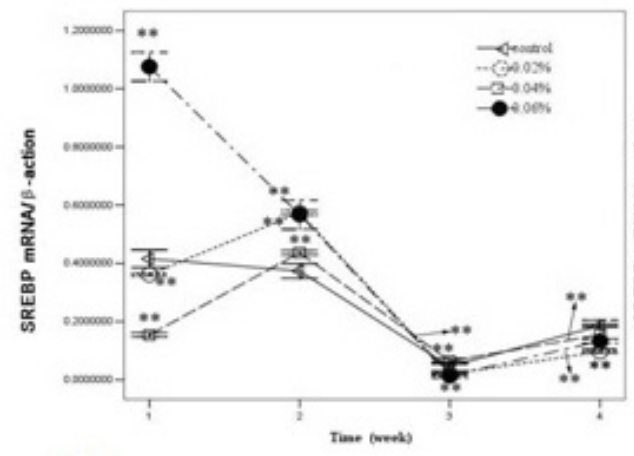

B

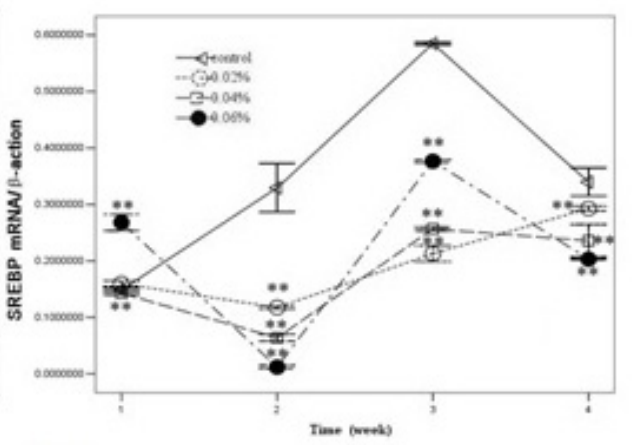

Figure 3. Evodiamine affects SREBP mRNA expression in mouse liver $(\mathbf{A})$ and adipose tissues $(\mathbf{B}) . * * \mathrm{P}<0.01$. 
A

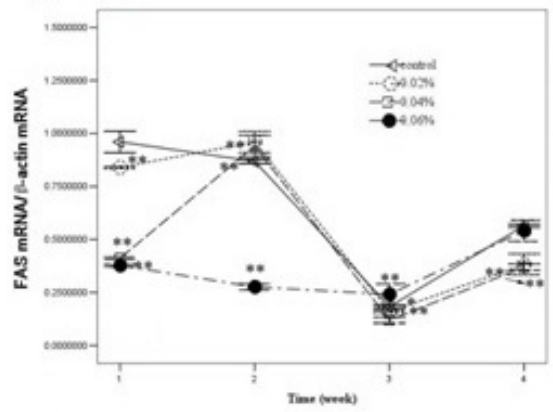

B

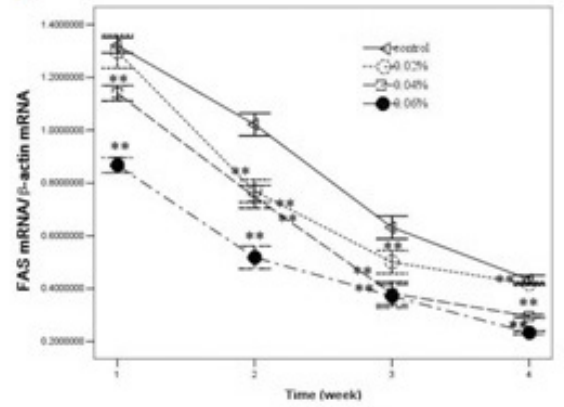

Figure 4. Effects of evodiamine on FAS mRNA expression in mouse liver (A) and adipose tissues $(\mathbf{B}) .{ }^{*} \mathrm{P}<0.01$.

\section{Effect of evodiamine on HSL and TGH expressions}

Given the above results, we investigated the effect that evodiamine might have on expressions of genes involved in lipid decomposition. The data showed that evodiamine increased the expression of HSL in both liver and adipose tissues (Figure 5A and B). Interestingly, no change in TGH expression was observed in liver tissues (Figure 6A), whereas it was significantly increased in adipose tissues in a time- and dose-dependent manner (Figure 6B).

A

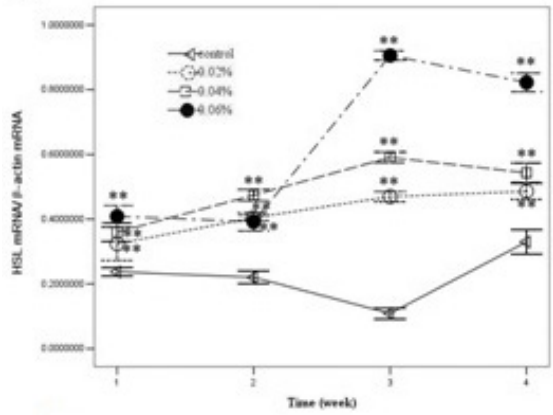

B

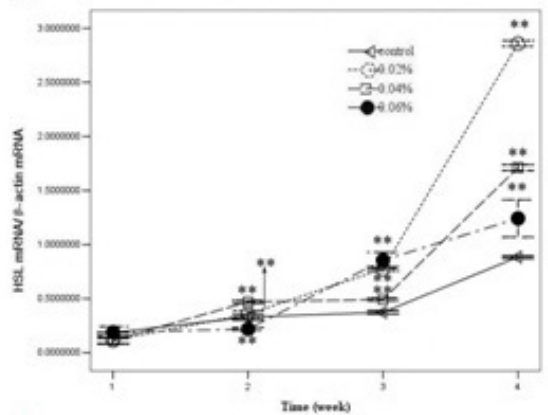

Figure 5. Evodiamine affects HSL expression in mouse liver $(\mathbf{A})$ and adipose tissues $(\mathbf{B})$. ${ }^{* *} \mathrm{P}<0.01$.

A

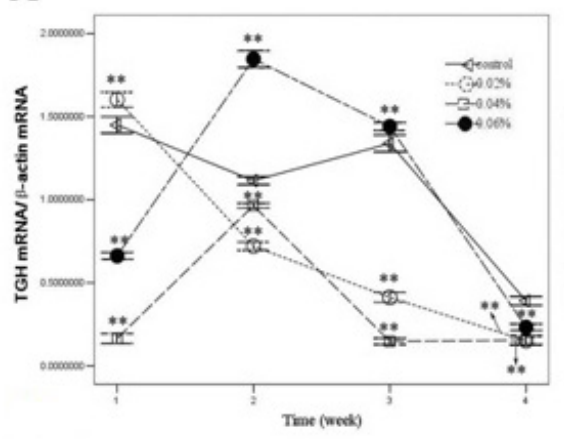

B

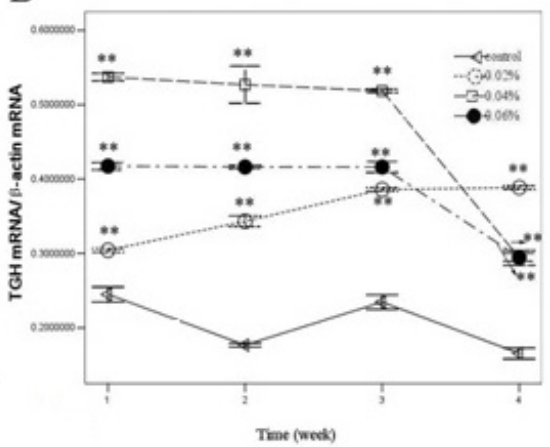

Figure 6. Evodiamine affects TGH expression in mouse liver $(\mathbf{A})$ and adipose tissues $(\mathbf{B})$. $* * \mathrm{P}<0.01$. 


\section{DISCUSSION}

Obesity is marked by excessive body fat and is closely related to lipid metabolic disorders, and the two reflect mutual causality. Lipid metabolic disorders can be caused by overeating, resulting in increased materials for fat synthesis and the reduction of brown fat content. In such cases, energy consumption is down-regulated, and deregulation of lipid-lowering hormone expressions induces fat synthesis and reduces its degradation. Holistic Chinese medicine theory suggests a slimming diet based on three core principles: weight loss without intensive physical exercise, appetite reduction without anorexia, and the absence of diarrhea. The integration of traditional Chinese medicine theories in modern biomedical technology has become an increasingly relevant concern.

In fat tissues, glucose and other nutrients are converted into fatty acids by the FASregulated pathway (Richards et al., 2003). Therefore, overexpression of FAS would increase triglyceride deposition in the body, which may cause obesity (Richards et al., 2003; Matsubara et al., 2005). Our results showed that FAS expression rapidly decreased upon evodiamine treatment in adipose tissues but not in the liver, indicating that this may be the main molecular mechanism underlying the effects of evodiamine on weight loss. SREBP-1c is an important transcription factor, which regulates the expression of FAS and lipoprotein lipase for fat synthesis (Kim and Spiegelman, 1996; Shimano et al., 1999). Therefore, as expected, we found that evodiamine rapidly reduced SREBP-1c expression in adipose tissues, whereas in the liver, for unknown reasons, SREBP-1c expression was increased.

PPAR $\gamma$ is considered an essential regulator in adipose tissues, which is closely correlated with fatty acid metabolism and triglyceride deposition. In adipocytes, PPAR $\gamma$ activates the expression of a variety of beneficial cell factors such as resistin, IL-6, and TNF $\alpha$ (Sharma and Staels, 2007; Qin et al., 2008), as well as plays multiple roles in glucose and lipid metabolism, atherosclerosis, and inflammatory responses (Tontonoz et al., 1995; Li et al., 2000; Fernyhough et al., 2007; Széles et al., 2007; Rampino et al., 2007). PPAR $\gamma$ is involved in the regulation of the expression of lipid metabolism-related genes, which can promote adipocyte differentiation, and a number of protein factor genes secreted by adipocytes, such as lipoprotein lipase, the fatty acid binding protein, acetyl CoA synthetase, and glucose transporter 4. PPAR $\gamma$ is a ligand-activating transcription factor, and once activated binds to the retinoic acid X receptor to form a heterodimer, which acts on DNA response elements to regulate the expressions of certain genes (Fajas et al., 1998; Yamauchi and Kadowaki, 2001). A couple of ligands are involved in PPAR $\gamma$ activation, which can be classified as synthetic and natural ligands. Both ligand types physically interact with the PPAR $\gamma$ protein to participate in the process of fat synthesis. Consequently, PPAR $\gamma$ has become a popular molecular target for the development of medications against obesity and diabetes. Compelling evidence has revealed that Chinese medicines, including single-flavor drugs, active ingredients, and compound preparations used for the treatment of obesity, all have effects on PPAR $\gamma$ expression (Huang et al., 2006; Chinetti-Gbaguidi et al., 2012). Therefore, it is somewhat surprising that we observed increases in PPAR $\gamma$ expression in adipose tissue (Figure 2B) and decreased expression levels in the liver (Figure 2A) as a result of evodiamine treatment. The reason might be 2-fold. First, evodiamine may act downstream of the PPAR $\gamma$ gene, and second, the increase of PPAR $\gamma$ might be a secondary effect due to the accumulation of free fatty acids and lipid metabolites in evodiamine-treated animals. Nevertheless, the observed changes in PPAR $\gamma$ and SREBP-1c ex- 
pressions upon evodiamine treatment in adipose and liver tissues were fairly rapid, indicating that these genes may be key players in fat synthesis, and are very sensitive to the presence of evodiamine. The opposite effect of evodiamine on these two genes in adipose and liver tissues may be due to complex regulatory mechanisms and differential biological effects of genes. In fact, the liver is the major site for energy consumption, whereas adipose tissue is involved in energy conservation in animals.

HSL and TGH are the major intracellular hydrolysis enzymes in adipose tissues, which can catalyze the hydrolysis of triglyceride to produce free fatty acids, thereby acting as an important energy source in vivo (Holm et al., 1988; Soni et al., 2004). The mRNA expressions of HSL and TGH in liver and adipose tissues increased significantly after evodiamine treatment, and the effects lasted for a very long period of time (data not shown). This result suggests that TGH and HSL might be the direct targets of evodiamine, and the regulation of lipid metabolism by evodiamine may involve increased expression of lipase mRNA.

Metabolic activities are accompanied by the release, metastasis, and utilization of energy. Two processes, with opposite effects, are involved in the flow of energy: catabolism is caving, while anabolism is needed for the supply of energy. The latter is also referred to as the "energy-absorbing reaction". In the present study, we found that evodiamine significantly affected the core body temperature of mice. Surprisingly, the decrease of body temperature by evodiamine was associated with increases of TGH and HSL mRNA expressions in both liver and adipose tissues. In contrast, increases in FAS and SREBP-1c mRNA expressions were only observed in the liver. In light of all the evidence obtained in this study, we conclude that the molecular mechanism by which evodiamine regulates body fat deposition is to reduce body temperature by raising energy consumption while simultaneously promoting fat decomposition.

\section{ACKNOWLEDGMENTS}

Research supported by grants from the National Nature Science Foundation of China (\#31172185).

\section{REFERENCES}

Chinetti-Gbaguidi G, Bouhlel MA, Copin C, Duhem C, et al. (2012). Peroxisome proliferator-activated receptor-gamma activation induces 11beta-hydroxysteroid dehydrogenase type 1 activity in human alternative macrophages. Arterioscler. Thromb. Vasc. Biol. 32: 677-685.

Fajas L, Fruchart JC and Auwerx J (1998). Transcriptional control of adipogenesis. Curr. Opin. Cell Biol. 10: 165-173.

Fernyhough ME, Okine E, Hausman G, Vierck JL, et al. (2007). PPARgamma and GLUT-4 expression as developmental regulators/markers for preadipocyte differentiation into an adipocyte. Domest. Anim. Endocrinol. 33: 367-378.

Holm C, Kirchgessner TG, Svenson KL, Fredrikson G, et al. (1988). Hormone-sensitive lipase: sequence, expression, and chromosomal localization to cent-q13.3. Science 241: 1503-1506.

Huang C, Zhang Y, Gong Z, Sheng X, et al. (2006). Berberine inhibits 3T3-L1 adipocyte differentiation through the PPARgamma pathway. Biochem. Biophys. Res. Commun. 348: 571-578.

Kim JB and Spiegelman BM (1996). ADD1/SREBP1 promotes adipocyte differentiation and gene expression linked to fatty acid metabolism. Genes Dev. 10: 1096-1107.

Ko HC, Wang YH, Liou KT, Chen CM, et al. (2007). Anti-inflammatory effects and mechanisms of the ethanol extract of Evodia rutaecarpa and its bioactive components on neutrophils and microglial cells. Eur. J. Pharmacol. 555: 211-217.

Kobayashi V, Nakano V, Kizaki M, Hoshikuma K, et al. (2001). Capsaicin-like anti-obese activities of evodiamine from fruits of Evodia rutaecarpa, a vanilloid receptor agonist. Planta Med. 67: 628-633.

Li AC, Brown KK, Silvestre MJ, Willson TM, et al. (2000). Peroxisome proliferator-activated receptor gamma ligands 
inhibit development of atherosclerosis in LDL receptor-deficient mice. J. Clin. Invest. 106: 523-531.

Matsubara Y, Sato K, Ishii H and Akiba Y (2005). Changes in mRNA expression of regulatory factors involved in adipocyte differentiation during fatty acid induced adipogenesis in chicken. Comp. Biochem. Physiol. A Mol. Integr. Physiol. 141: 108-115.

Ogasawara M, Matsunaga T, Takahashi S, Saiki I, et al. (2002). Anti-invasive and metastatic activities of evodiamine. Biol. Pharm. Bull. 25: 1491-1493.

Qin X, Xie X, Fan Y, Tian J, et al. (2008). Peroxisome proliferator-activated receptor-delta induces insulin-induced gene-1 and suppresses hepatic lipogenesis in obese diabetic mice. Hepatology 48: 432-441.

Rampino T, Ranghino A, Guidetti C, Gregorini M, et al. (2007). Activation of PPARgamma enhances in vitro the immunosuppressive effect of cyclosporine on T lymphocytes. Transpl. Immunol. 18: 32-36.

Richards MP, Poch SM, Coon CN, Rosebrough RW, et al. (2003). Feed restriction significantly alters lipogenic gene expression in broiler breeder chickens. J. Nutr. 133: 707-715.

Sharma AM and Staels B (2007). Review: Peroxisome proliferator-activated receptor gamma and adipose tissue understanding obesity-related changes in regulation of lipid and glucose metabolism. J. Clin. Endocrinol. Metab. 92: 386-395.

Shimano H, Yahagi N, Amemiya-Kudo M, Hasty AH, et al. (1999). Sterol regulatory element-binding protein-1 as a key transcription factor for nutritional induction of lipogenic enzyme genes. J. Biol. Chem. 274: 35832-35839.

Shyu KG, Lin S, Lee CC, Chen E, et al. (2006). Evodiamine inhibits in vitro angiogenesis: Implication for antitumorgenicity. Life Sci. 78: 2234-2243.

Soni KG, Lehner R, Metalnikov P, O’Donnell P, et al. (2004). Carboxylesterase 3 (EC 3.1.1.1) is a major adipocyte lipase. J. Biol. Chem. 279: 40683-40689.

Széles L, Töröcsik D and Nagy L (2007). PPAR $\gamma$ in immunity and inflammation: cell types and diseases. Biochim. Biophys. Acta 1771: 1014-1030.

Tontonoz P, Hu E and Spiegelman BM (1995). Regulation of adipocyte gene expression and differentiation by peroxisome proliferator activated receptor gamma. Curr. Opin. Genet. Dev. 5: 571-576.

Wang T, Wang Y, Kontani Y, Kobayashi Y, et al. (2008). Evodiamine improves diet-induced obesity in a uncoupling protein-1-independent manner: involvement of antiadipogenic mechanism and extracellularly regulated kinase/ mitogen-activated protein kinase signaling. Endocrinology 149: 358-366.

Yamauchi T and Kadowaki T (2001). The molecular mechanisms by which PPAR gamma/RXR inhibitors improve insulin resistance. Nihon Rinsho 59: 2245-2254. 